\title{
Take Shuibu Town of Taishan city as an example to study the application of GIS in small towns under the background of "Muti- planning integration"
}

\author{
Yulu Liao $^{1}$ \\ ${ }^{1}$ School of Civil Engineering and Architecture, Wuhan University of Technology, Wuhan, Hubei, 430070, China
}

\begin{abstract}
With the proposal of land planning strategy and the development of GIS information technology and big data,geographic information technology and big data are widely used in the field of urban and rural planning,providing new technical support for the implementation of "muti-planning integration". As the reserve force for development,small towns have great research value.This study selects Shuibu Town of Taishan city to study the construction and management of Urban Land GIS database in order to provide working ideas for the construction of GIS data platform in small towns.
\end{abstract}

\section{Introduction}

Recently, with the high-quality development of social economy and the continuous advancement of ecological civilization construction, the establishment of spatial development planning system and the unification of territorial space use control have become the current research hotspots. As the drawings used by each resource planning are not uniform, conflicts between multiple professional planning arise. In order to better integrate resource data of economic society, land, planning, forestland, arable land, transportation and other aspects, and form a unified information management platform, "muti-planning integration" emerges at the right moment. The ultimate purpose of land space planning is also to achieve "muti-planning integration"[1].

Small towns located in urban megalopolis are characterized by relatively high land use intensity, abundant natural resources, complex internal elements and lack of effective management of spatial elements, etc., which often become the objects of urban competition for land resources ${ }^{[2]}$. The study of "muti-planning integration" in small towns within metropolitan areas can not only promote the development of small towns themselves, but also exert a positive influence on the development of large cities around them.

GIS is a technology that uses computers for the comprehensive application of multiple disciplines, and it is mainly used for the input and output of geographic data, management and control, collation, search, inquiry, analysis and decision making of computer data ${ }^{[3]}$. In the process of "multi-planning integration", each planning has a large amount of graphic or non-graphic data. GIS technology can be used to integrate multi-source data, build a unified information database, and lay a good foundation for "one map" planning and visually analyze and express the collected data.

\section{Basic data collection and collation}

\subsection{The date collection}

The data used in this study include GIS data of the third National Land Survey of Shuibu Town in 2018 (hereinafter referred to as "the third Survey"), DEM data of Taishan City, CAD data of Shuibu Town in 2013, social and economic statistics data of all towns and villages released by the People's government of Shuibu Town in 2018, and high-definition satellite image map in 2020.

\subsection{Data collation based on GIS}

\subsubsection{Organize and build database of current data}

(1) The registration of GIS data with satellite images and the approval of GIS data combined with satellite images.

(2) Derived from the CAD data with elevation point $Z$ coordinate properties, and save as new low version CAD file. And then, match the CAD file with the GIS data.

(3) The social and economic data such as the name of each village and the total population of each village published by the People's government of Shuibu Town are shown in the Excel. Then, the GIS data of Shuibu town are fused according to the field of village name. Finally, connect the Excel table to a new SHP file named "Village Fusion"

\subsubsection{Status statistics of land use in Shuibu Town}

According to the classification of the third National Land Survey, the land is divided into 12 first-level land types

\footnotetext{
*Corresponding author: yululiao@whut.edu.cn
} 
and 73 second-level land types by "the third Survey", while the land for territorial space planning is divided into 28 first-level land types, 102 second-level land types and 39 third-level land types. Therefore, the land classification of the GIS data in "the third survey" needs to be classified and converted according to the Guidelines for Basic Classification and Use Classification of Urban and County Land Space Planning (trial version, solicitation draft).

There are four kinds of situations in the transformation of "the third survey" land classification: "one-to-one correspondence", "one-to-many","many-to-one"and "noncorrespondence".

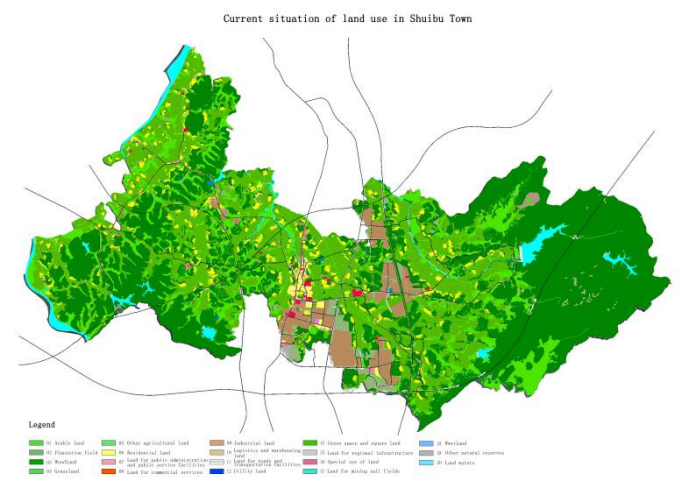

Fig. 1. Current situation of land use in Shuibu Town

After the deal with the land use classification, open GIS data in Shuibu attribute table to create a new field, use field calculator, write a code block, dispatch land use classification in "the third survey" according to the above method into the corresponding land use classification. And then,using integration tools, the fields are fused according to the new land classification to obtain a land classification layer according to the land planning. Finally, the legend is changed to derive the land use status map (See Figure 1).

\section{Thematic research based on GIS}

\subsection{Analysis of social and economic situation}

Open the property sheet on the "Village Fusion" SHP layer connected with the socio-economic Excel table, create a new "Area" field, conduct geometric calculation, and get the area of each village.Next, create a new field and use the field calculator to calculate the population density of each village. Finally, right-click on the layer and change the "levels" in the properties to get a visual representation of the population density of each village.

Create a new layer after village fusion and name it "Village Fusion 2". Connect the new layer to the Excel table, select the "population" field to represent the histogram in the properties, then change the color of the histogram and add 3D effects in the Settings (See Figure 2).

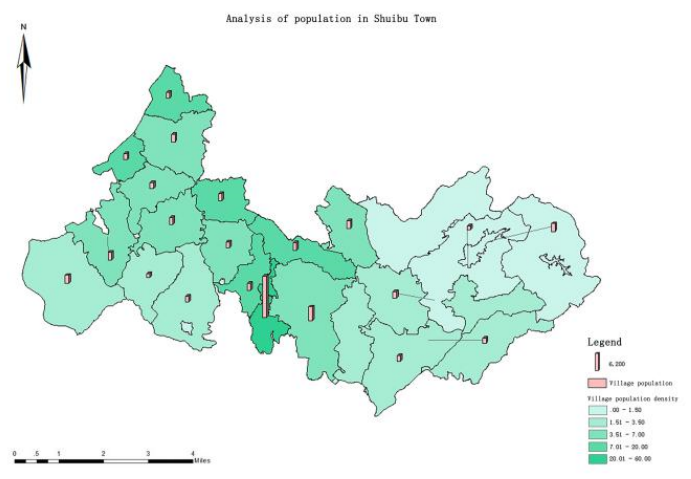

Fig. 2. Analysis of population in Shuibu Town

\subsection{Evaluation of ecological sensitivity}

\subsubsection{Impact factor analysis}

Topographic and topographic factors mainly refer to slope, aspect, elevation and so on. Slope can be divided into three grades according to the requirements of slope suitability for urban construction land. Generally speaking, the slope below $8.5^{\circ}$ can be regarded as suitable for urban construction, $8.5^{\circ}--11.3^{\circ}$ is the secondary suitable construction area, and more than $14^{\circ}$ is the inappropriate construction area ${ }^{[4]}$.

The impact factors of water area are mainly divided into streams and their affected areas, large reservoirs or irrigation channels and others.Because of the factors to improve the quality of urban landscape waters, adjust temperature humidity and improve the urban space environment plays an important role, and easy to cause floods. Therefore, the development and construction of land should be kept away from the water as far as possible so as not to cause damage to the aquatic ecosystem.So, from the perspectives of pollution sensitivity, landscape effect, wildlife habitat and so on, the ecological sensitivity of water factors was determined to be the highest, followed by large reservoirs and irrigation channels.

Among various types of land use, basic farmland belongs to the land category strictly controlled by the state and is rigidly protected by national policies and regulations. The industrial land will produce noise, dust, waste gas and waste water pollution, which has a great impact on the living environment and ecological environment, so it should be included in the sensitivity evaluation system. Shuibu Town is a town with agriculture as its foundation and industry as its main development target in the near future. It is mainly evaluated from its scope of influence.

The terrain topography, water area factor, basic farmland and industrial land were scored by analytic hierarchy process, and each weight was obtained (See Table 1). 
Table 1. Impact factor weight

\begin{tabular}{|c|c|c|c|c|c|c|c|c|}
\hline 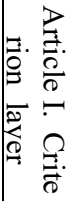 & \multicolumn{3}{|c|}{ 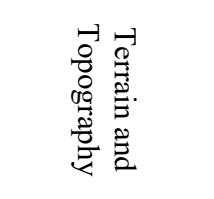 } & \multicolumn{3}{|c|}{ 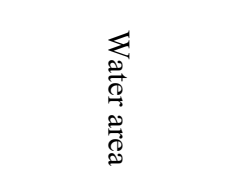 } & 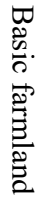 & 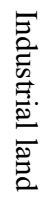 \\
\hline 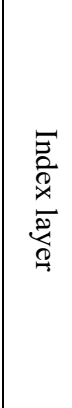 & 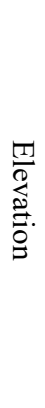 & $\frac{n}{0}$ & $\begin{array}{l}\text { D } \\
\underset{0}{0} \\
\stackrel{8}{\Im}\end{array}$ & 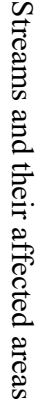 & 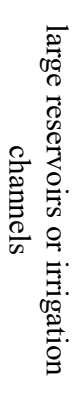 & 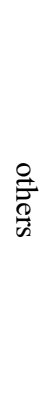 & 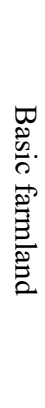 & 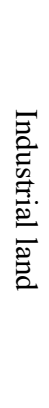 \\
\hline 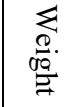 & $\stackrel{\circ}{9}$ & $\stackrel{\circ}{\dot{N}}$ & ن & $\stackrel{\stackrel{0}{\tilde{o}}}{ }$ & $\stackrel{0}{\not}$ & $\stackrel{\circ}{\infty}$ & $\stackrel{\stackrel{O}{\sim}}{\mathrm{w}}$ & 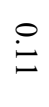 \\
\hline
\end{tabular}

\subsubsection{Impact factor evaluation}

Make the elevation point "Annonation" layer into a Tin, and convert the Tin into a grid to get the elevation grid. Then "Slope Analysis" and "Aspect Analysis" were used to obtain the slope analysis diagram and slope direction analysis diagram . Finally, reclassification is used to assign values to different terrain conditions for weighted stacking.

The technical route of ecological sensitivity assessment of water area, basic farmland and industrial land is similar to the terrain and topography, and the special map can be derived through GIS. After the influence factor analysis chart of all assigned values was obtained, the weighted sum was used to

obtain the ecological sensitivity evaluation chart of Shuibu Town (Figure 3). The higher the score was, the higher the ecological sensitivity was, and the lower the score was.

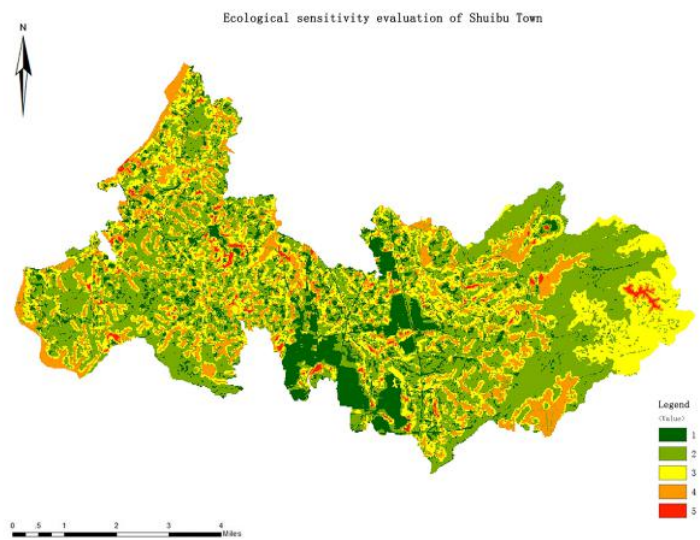

Fig. 3. Ecological sensitivity evaluation analysis chart

\subsection{Analysis of service facility configuration}

Firstly, based on the analysis of the current situation of Shuibu Town, seven influencing factors are extracted: hospitals, kindergartens, primary schools, middle schools and adult technical schools, garbage stations, public toilets and supermarkets. The weights of each factor are obtained through the analytic hierarchy process (See Table 2).

Table 2. Service facility configuration weights

\begin{tabular}{|c|c|c|c|c|c|c|c|}
\hline 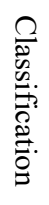 & 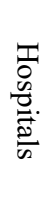 & 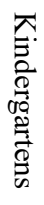 & 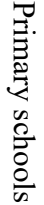 & 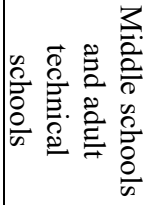 & 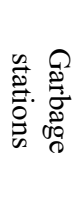 & 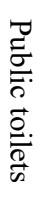 & 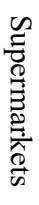 \\
\hline$\underset{\substack{0 \\
\stackrel{0}{0} .}}{\sum_{0}}$ & $\stackrel{\circ}{\sim}$ & io & $\stackrel{0}{a}$ & $\stackrel{\circ}{\dot{N}}$ & $\begin{array}{l}0 \\
\dot{0} \\
\text { Ui }\end{array}$ & $\stackrel{0}{0}$ & $\stackrel{0}{\text { i }}$ \\
\hline
\end{tabular}

And then, create seven new SHP layers and compare them with the satellite map. Edit the SHP elements on different layers. Then, the multi-ring buffer zone was established according to the corresponding service radius of different service facilities, and then the multi-ring buffer zone was connected with the "the third survey" GIS data for fusion. After that, the fused data of different service facilities were turned into grids. Finally, the raster data were reclassified and weighted and superimposed to obtain the analysis diagram of service facility configuration (See Figure 4).

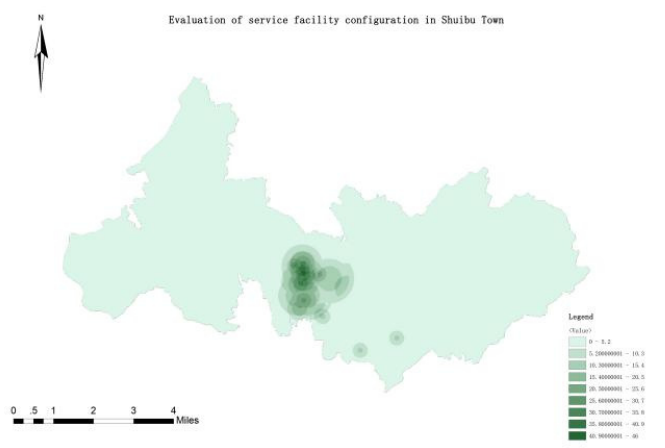

Fig. 4. Service facility configuration evaluation chart

\section{Conclusion}

With the help of Arcgis powerful data calculation and spatial analysis using geographic conditions survey data, combined with the relevant national technical specifications, this research makes an attempt evaluate the social resources, ecological sensitivity and service facility allocation in Shuibu Town, which not only reflects the application direction of the census data of geographical conditions, but also reflects the role of GIS in land space planning.

Before the concept of "muti-planning integration" was formally proposed, the community has been experimenting with multiple data superpositions. However, the practice of "muti-planning integration" in big cities is still at the exploratory stage, while the practice of "muti-planning integration" in small towns is very few.

Compared with big cities, small towns have many 
problems, such as imperfect basic data, lack of scientific data management, high complexity and heavy workload in data processing. This paper attempts to explore the small town GIS geographic data management platform and uses GIS to analyze Shuibu Town, in order to provide feasible ideas for the planning of small towns.

\section{References}

1. He Hong,Shi Zhiwei. Survey World, 4,69-86(2020)

2. Xiao Shaoying, Sha Shiyan,Bian Guangmeng,Li Weihua. Housing and Real Estate, 11,208-208(2019)

3. Wu Xincai. Earth Science -- Journal of China University of Geosciences, 23,229-333 (1998)

4. Zhou Yijun,Zhou Wei,Wang Huimin. Geomatics \& Spatial Information Technology, 41,97-105(2018) 